\title{
Uso de ossos bovinos calcinados como aditivo de sinterização na fabricação de porcelanas
}

\section{(Bone ash as a sintering additive in the porcelain production)}

\author{
D. Gouvêa ${ }^{1}$, T. T. Kaneko ${ }^{1}$, H. Kahn ${ }^{2}$ \\ ${ }^{l}$ Laboratório de Processos Cerâmicos, Departamento de Engenharia Metalúrgica e de Materiais \\ ${ }^{2}$ Laboratório de Caracterização Tecnológica, Departamento de Engenharia Minas e Petróleo \\ Escola Politécnica da Universidade de S. Paulo - EPUSP, Av. Prof Mello Moraes 2463, Cidade Universitária, \\ S. Paulo, SP 05580-900 \\ dgouvea@usp.br
}

\begin{abstract}
Resumo
Ossos bovinos calcinados (OBC) são geralmente utilizados para a fabricação de uma porcelana de alta qualidade conhecida como bone china. Nesses produtos a quantidade de OBC é cerca de $50 \%$ do produto. Apesar de o rebanho bovino brasileiro ser um dos maiores do mundo com cerca de 200 milhões de cabeças, grande parte dos resíduos de ossos ainda não é aproveitada. Contudo, é conhecido que o $\mathrm{OBC}$ em pequenas quantidades e associado com materiais-primas fundentes, como feldspatos, pode reduzir a temperatura de liquidus e com isso promover a sinterização via fase líquida. A finalidade deste trabalho foi de verificar a potencialidade do uso de OBC como promotor da sinterização de porcelanas fabricadas com o sistema triaxial clássico: caulim, feldspato e quartzo. Foram introduzidas quantidades de 1,2 e 5\% em massa e sinterizadas entre 1100 e $1350{ }^{\circ} \mathrm{C}$. As amostras foram comparadas com a formulação sem adição de $\mathrm{OBC}$ com respeito à limite de resistência e absorção de água, formação de fases por difração de raios X e observações no microscópio eletrônico de varredura. A temperatura de sinterização foi reduzida em $50{ }^{\circ} \mathrm{C}$ para a quantidade de $2 \%$ de $\mathrm{OBC}$, porém grande quantidade de porosidade residual pode ser gerada se houver aquecimento demasiado das porcelanas.

Palavras-chave: porcelana de ossos.
\end{abstract}

\begin{abstract}
Bone ash is typically used as raw material to produce high quality porcelain denoted bone china. The total amount of bone ash is around 50\%. With about 200 million head, Brazil has the largest commercial herd of cattle in the world but the bovine bones are not employed in the Brazilian ceramic industry. Nevertheless, additions of bone ash in the raw material composition used in the porcelain fabrication reduce the liquidus temperature and could be an additive for liquid phase sintering. In this work, bone ash was used as a sintering additive in the fabrication of classic porcelain: kaolin, feldspar and quartz. Porcelain was produced containing bone ash $(0,1,2$ and $5 \%)$ and sintered from 1100 to $1350^{\circ} \mathrm{C}$. Water absorption and modulus of rupture were measured and related with the microstructure observation by $M E V$ and phase formation by X-ray diffraction. The sintering temperature was reduced by $50{ }^{\circ} \mathrm{C}$ for $2 \%$ of bone ash while the mechanical strength was increased. A high residual porosity was observed for sample with over firing.

Keywords: bone china.
\end{abstract}

\section{INTRODUÇÃO}

Porcelana é um material cerâmico constituído de óxidos do sistema triaxial alumina-sílica-óxido de metais alcalinos (principalmente $\mathrm{K}_{2} \mathrm{O}$ ) e é fabricada a partir de uma mistura básica de caulim, feldspato e quartzo próxima de composição 50:25:25, respectivamente [1-3]. Parte do caulim pode ser substituída por argilas plásticas com a função de aumentar a resistência mecânica a cru e a plasticidade das massas. As porcelanas apresentam como características únicas: grande resistência mecânica, temperaturas de queima acima de $1350{ }^{\circ} \mathrm{C}$, porosidade abaixo de $0,3 \%$ e elevadas alvura e translucidez [4]. Levando-se em conta todas estas características, não existe produto comercial nacional que possa ser defino como "porcelana" . O fundente geralmente utilizado é o feldspato na forma de albita ou ortoclásio, que contém respectivamente maior teor de óxido de sódio e de potássio. Outras matérias-primas fundentes são possíveis e podemos destacar: os filitos, os pegmatitos e mais recentemente os fonolitos [5].

As diferentes proporções podem modificar a temperatura de queima e a resistência final dos produtos e, geralmente, o feldspato utilizado para a porcelana é principalmente o ortoclásio [4].

Os fundentes utilizados em porcelana são muito restritos devido às características particulares da fase vítrea formada 
durante a queima desses materiais. Como as porcelanas apresentam cerca de $70 \%$ de fase vítrea e $30 \%$ de fase cristalina, a viscosidade da fase vítrea e a quantidade de fase cristalina controlam a resistência desses materiais à deformação piroplástica. Calcita e dolomita podem ser utilizadas para acelerar a fusão dos feldspatos e reduzir a temperatura de queima sem modificar significativamente a deformação piroplástica. Contudo, estes carbonatos têm como principal inconveniente a formação de $\mathrm{CO}_{2}$ como produto da sua decomposição em temperaturas superiores a $900{ }^{\circ} \mathrm{C}$. Para materiais cerâmicos produzidos por monoqueima ou ciclos térmicos rápidos a formação de gases em temperaturas elevadas pode gerar defeitos que prejudicam o desempenho dos produtos finais.

Uma alternativa para acelerar a fusão dos feldspatos pela introdução de fosfato de cálcio pode ser a utilização de ossos bovinos calcinados. O uso de ossos bovinos calcinados (OBC) é restrito à fabricação de "bone china" e a quantidade OBC é cerca de 50\% do total da matéria-prima [1]. As porcelanas de osso bovino são mais resistentes, translúcidas e alvas que as porcelanas de caulim [6], contudo não existe produção desse material na América Latina. O OBC é basicamente hidroxiapatita, que em combinação com caulim e feldspato pode formar vidros. A porcelana bone china é um material que contém aproximadamente $30 \%$ de fase amorfa e $70 \%$ de fase cristalina e a fase vítrea é rica em sílica, mas contém também $\mathrm{Al}_{2} \mathrm{O}_{3}, \mathrm{~K}_{2} \mathrm{O}$ e $\mathrm{CaO}$ [1]. O uso de OBC pode levar à modificação da temperatura de liquidus [7-9] do sistema e reduzir a temperatura de sinterização que ocorre via fase líquida.

A proposta deste trabalho é verificar a influência do OBC em quantidades inferiores a 5\% em massa na queima de uma massa cerâmica de porcelana industrial. Os produtos da queima das porcelanas em temperaturas no intervalo de 1000 a $1350{ }^{\circ} \mathrm{C}$ e contendo $0,1,2$ e $5 \%$ em massa de OBC foram avaliados através do acompanhamento da absorção de água e da resistência mecânica à flexão. A decomposição e/ou formação de fases foi realizada através da técnica de difração de raios X e as informações da microestrutura foram obtidas através da observação dos materiais utilizando-se um microscópio eletrônico de varredura.

\section{MATERIAIS E MÉTODOS}

A massa de porcelana utilizada foi preparada com uma composição próxima a padrão, ou seja, $50 \%$ caulim, $25 \%$ ortoclásio e $25 \%$ quartzo e cedida pela empresa Porcelanas Teixeira. A massa moída em moinho industrial foi seca e nesta foram introduzidas as quantidades de 1,2 e $5 \%$ em massa de $\mathrm{OBC}$ e a mistura foi executada em moinho de bolas de laboratório por $24 \mathrm{~h} \mathrm{e} \mathrm{com} \mathrm{elementos} \mathrm{de} \mathrm{moagem} \mathrm{de}$ porcelana. O produto foi disperso utilizando um poliacrilato de sódio industrial e os corpos de prova com tamanho padrão de $80 \times 30 \times 3 \mathrm{~mm}^{3}$ foram obtidos por colagem de barbotina em moldes de gesso.

O osso de boi calcinado foi preparado segundo descrito em trabalhos anteriores $[10,11]$. O material submetido a autoclave foi calcinado a $700{ }^{\circ} \mathrm{C}$ e moído em moinho de bolas. Os corpos de prova foram submetidos ao tratamento térmico em forno tipo mufla e aquecidos a $2{ }^{\circ} \mathrm{C} / \mathrm{min}$ até a temperatura de sinterização e desligado para resfriamento natural sem qualquer patamar de temperatura. As temperaturas utilizadas foram entre 1000 e $1350{ }^{\circ} \mathrm{C} \mathrm{com}$ intervalos de $50{ }^{\circ} \mathrm{C}$. As peças produzidas foram submetidas a teste de resistência mecânica 3 pontos em máquina universal Kratos com velocidade de $6 \mathrm{~mm} / \mathrm{min}$ e distância dos apoios de $40 \mathrm{~mm}$. A absorção de água foi medida em cada amostra inicialmente determinando a massa seca a $110^{\circ} \mathrm{C}$ e depois da massa úmida após fervida em água durante $2 \mathrm{~h}$ e resfriada.

Para medidas de difração de raios $\mathrm{X}$ as amostras foram moídas em almofariz de ágata para evitar problemas de orientação preferencial das partículas cristalinas formadas durante a queima. O equipamento utilizado foi Bruker-AXS modelo D8 Advance operando com radiação $\mathrm{Cu}-\mathrm{k}_{\alpha}$, com passo $2 \theta$ de $0,02^{\circ}$ e tempo de contagem por passo $5 \mathrm{~s}$.

Observações no microscópio eletrônico de varredura foram feitas em amostras cortadas, lixadas e polidas segundo as técnicas convencionais de ceramografia em equipamento Leica Cambridge, Stereoscan 440, com tensão de aceleração de $10 \mathrm{kV}$.

\section{RESULTADOS E DISCUSSÃO}

Os resultados de absorção de água (AA) das amostras sinterizadas com $0,1,2$ e 5\% de OBC são apresentados na Fig. 1. É possível observar que dois fenômenos ocorrem simultaneamente quando se introduzem maiores quantidades de OBC: primeiro a diminuição da temperatura para fechamento total dos poros, e segundo a diminuição do intervalo de queima, ou seja, o intervalo de temperatura entre a porosidade completamente aberta para fechada. Contudo, a observação das amostras polidas no MEV revela que, apesar das cerâmicas apresentarem baixa absorção de água, existe a formação de grande porosidade fechada (Fig. 2). O

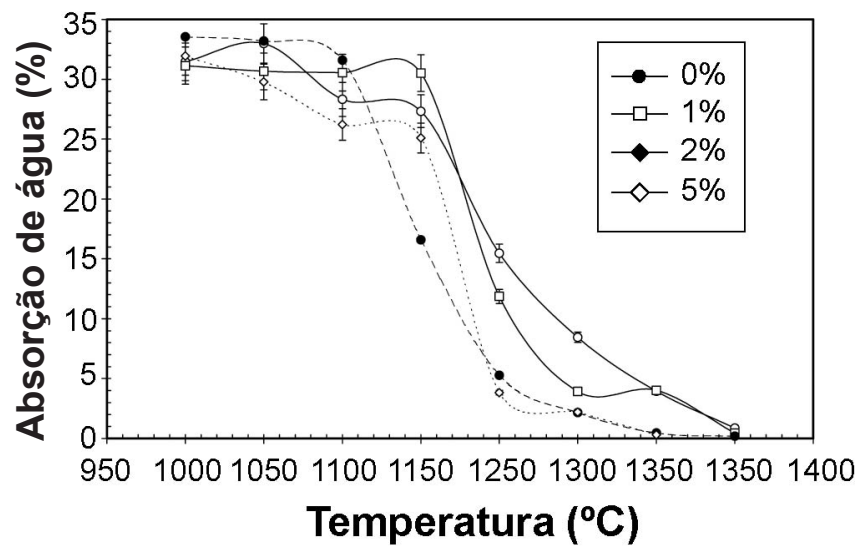

Figura 1: Curvas de absorção de água de amostras de porcelana contendo diferentes quantidades de OBC em função da temperatura de sinterização.

[Figure 1: Water absorption of porcelain samples containing bone ash $(0,1,2$ and $5 w t \%)$ and sintered at different temperatures.] 


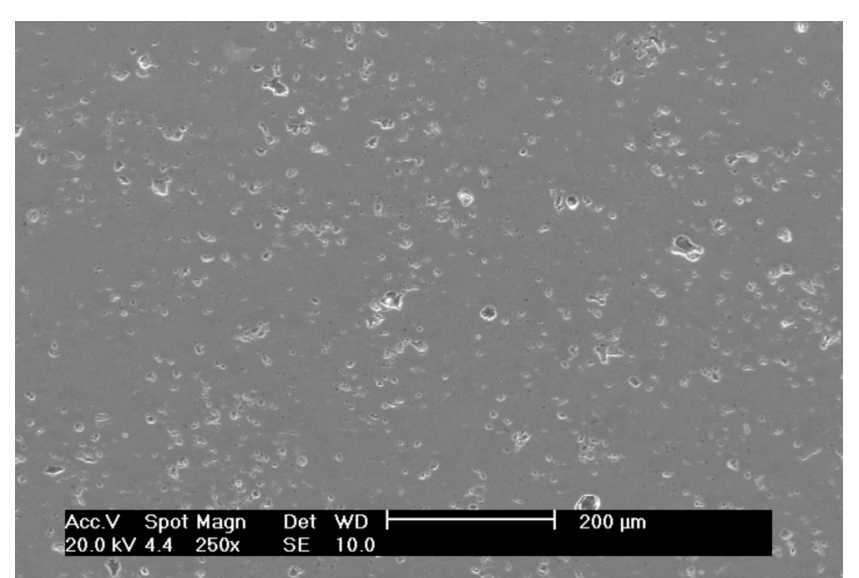

(a) $0 \% 1350^{\circ} \mathrm{C}$

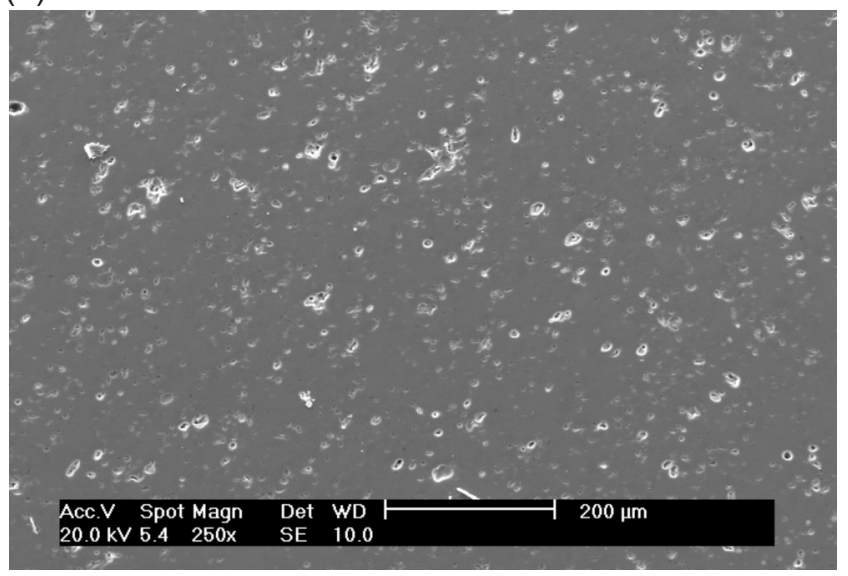

(b) $1 \% 1350{ }^{\circ} \mathrm{C}$

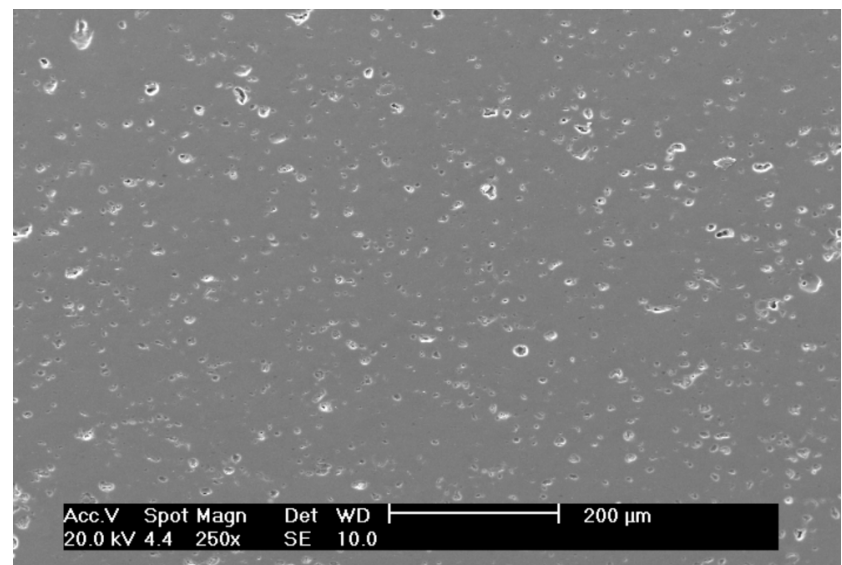

(d) $2 \% 1300^{\circ} \mathrm{C}$

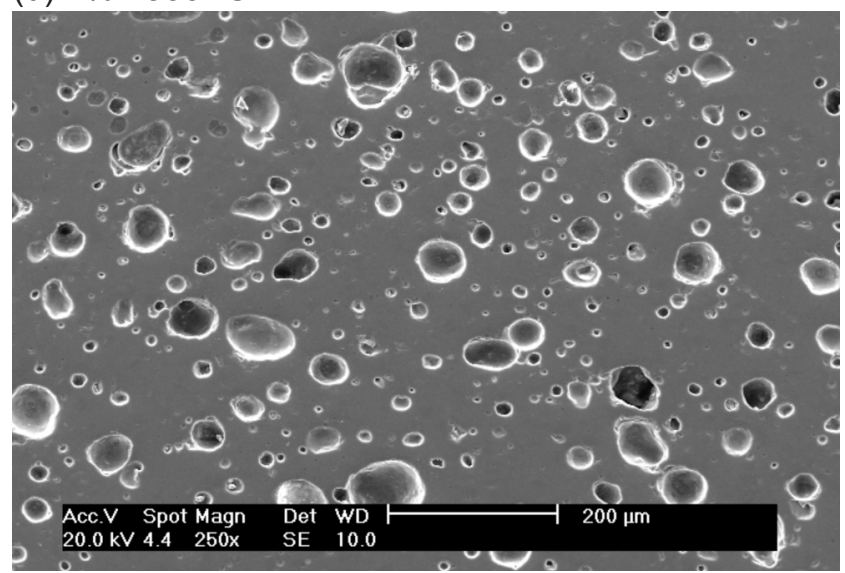

(e) $5 \% 1300{ }^{\circ} \mathrm{C}$

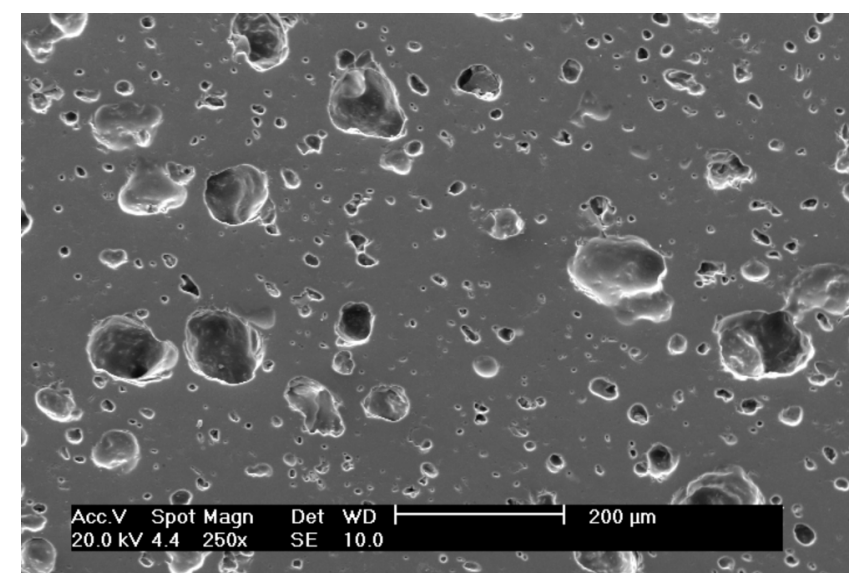

(c) $2 \% 1350^{\circ} \mathrm{C}$

Figura 2: Micrografias de amostras de porcelana contendo diferentes quantidades de OBC e sinterizadas em várias temperaturas. [Figure 2: SEM micrographs of porcelain samples containing different amounts of bone ash.]

inusitado neste caso é que a porosidade é formada após uma determina temperatura, antes da qual não havia porosidade residual (Figs. 2c e 2d). Isto se deve provavelmente à eliminação de algum produto gasoso ou mesmo vapor de água e deve ser proveniente do $\mathrm{OBC}$ introduzido. O aumento da quantidade de OBC para materiais queimados numa mesma temperatura também demonstra claramente que a formação do produto gasoso tem uma relação direta entre a quantidade de $\mathrm{OBC}$ e a temperatura de queima, como pode ser observado na seqüência de micrografias das Figs. 2a a 2c. Quando a queima é realizada a $1350{ }^{\circ} \mathrm{C}$ para a amostra com $1 \%$ de OBC (Fig. 2b), a porosidade residual é semelhante à amostra sem OBC (Fig. 2a). Contudo, mesmo mantendo a temperatura de queima, a adição de $2 \%$ de OBC leva a uma formação significativa de poros arredondados e fechados no material (Fig. 2c). A forma arredondada dos poros também é um indício de que o vapor ou gás formado durante a queima está imerso em uma fase vítrea de baixa 
viscosidade. O aumento da quantidade de fase vítrea pode ser avaliado pela comparação dos difratogramas de raios $\mathrm{X}$ (Fig. 3). Tomando-se o cuidado de utilizar a raia mais intensa do quartzo como padrão, ou seja, igualando as amplitudes dos picos mais intensos do quartzo das análises dos materiais contendo adição de 0 e $5 \%$ de $\mathrm{OBC}$ e sinterizadas a $1350{ }^{\circ} \mathrm{C}$. Fica evidente que a banda que surge devido à formação da fase vítrea encontrada entre 15 e $35^{\circ}$ tem sua intensidade aumentada significativamente quando comparada aos picos de quartzo, demonstrando que existe, pelo menos do ponto de vista qualitativo, um incremento na quantidade de fase vítrea.

A observação dos difratogramas da Fig. 3 também mostra que não existe mudança das fases cristalinas nos materiais

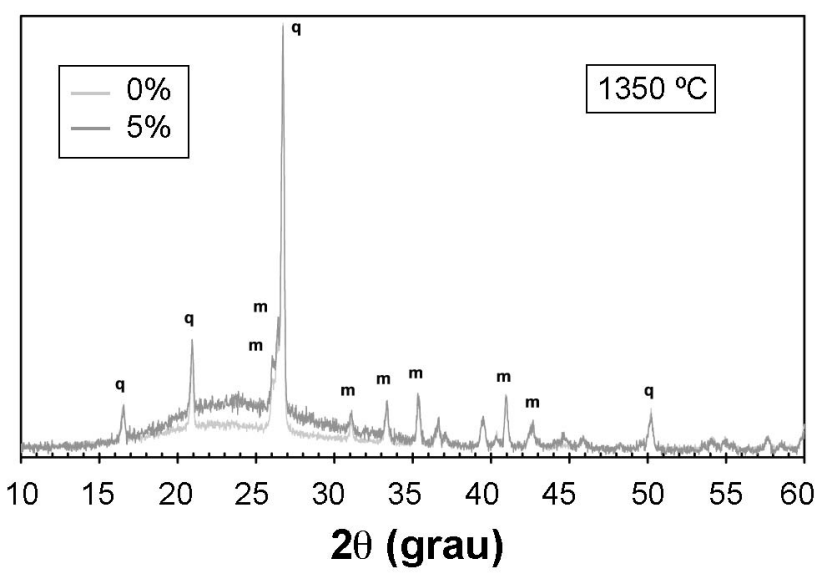

Figura 3: Difratogramas de raios $\mathrm{X}$ de duas amostras de porcelana: uma sem adição de OBC e a segunda com adição de 5\% de OBC e sinterizadas a $1350{ }^{\circ} \mathrm{C}(\mathrm{q}=$ quartzo e $\mathrm{m}=$ mulita $)$

[Figure 3: XRD patterns of two porcelain samples sintered at $1350{ }^{\circ} \mathrm{C}$ containing $5 \mathrm{wt} \%$ of bone ash.]

com diferentes quantidades de OBC. As fases encontradas, além da fase vítrea, foram: mulita, formada a partir da decomposição das matérias-primas durante a queima, e quartzo residual. Estas fases são comuns à porcelana sinterizadas nos intervalos de temperatura utilizados e nenhuma fase cristalina adicional, que poderia surgir a partir da reação do OBC com as matérias-primas, foi identificada nos difratogramas.

A formação das fases, tanto a líquida em maior quantidade para maior quantidade de OBC, como a gasosa confinada na porosidade fechada, deve ter um papel fundamental no comportamento mecânico do material final. A avaliação da resistência à fratura das amostras foi realizada e os resultados apresentados na Fig. 4.

O comportamento mecânico reflete em muito a evolução microestrutural dos corpos cerâmicos. $\mathrm{O}$ aumento da porosidade fechada representa um decréscimo da resistência mecânica. Contudo, é muito importante destacar que a amostra contendo $2 \%$ de $\mathrm{OBC}$ e queimada a $1300{ }^{\circ} \mathrm{C}$ apresentou microestrutura semelhante à amostra com $0 \% \mathrm{e}$ queimada a $1350{ }^{\circ} \mathrm{C}$ e uma resistência mecânica superior.

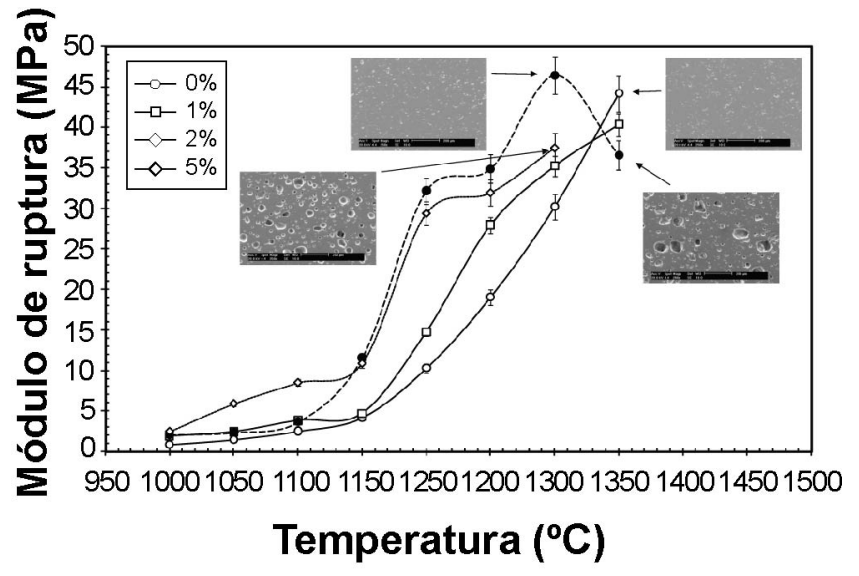

Figura 4: Módulo de ruptura das amostras de porcelana contendo 0 , 1,2 e $5 \%$ de $\mathrm{OBC}$ e sinterizadas de 1000 a $1350{ }^{\circ} \mathrm{C}$ com intervalos de $50{ }^{\circ} \mathrm{C}$. Detalhes das microestruturas geradas para algumas composições e temperaturas.

[Figure 4: Rupture modulus of porcelain samples containing 0, 1,2 and $5 \mathrm{wt} \%$ of bone ash and sintering between 1000 and $1350^{\circ} \mathrm{C}$.]

Isto mostra que a introdução do $\mathrm{OBC}$ reduz a temperatura de queima das porcelanas e aumenta a resistência mecânica, desde que utilizado em temperaturas e quantidades adequadas.

\section{CONCLUSÕES}

A introdução de osso bovino calcinado (OBC) em uma massa de porcelana comercial diminui a temperatura de queima devido ao aumento da quantidade de vidro formado e facilitando a sinterização. Nenhuma nova fase é detectada, somente um aumento significativo da fase vítrea. Contudo a formação de defeitos na forma de bolhas ocorre para determinadas quantidades de $\mathrm{OBC}$ e temperatura de sinterização. Os defeitos diminuem a resistência mecânica da porcelana. Porcelanas contendo $2 \%$ de OBC foram densificadas em temperaturas de $50{ }^{\circ} \mathrm{C}$ inferiores que porcelanas sem $\mathrm{OBC}$ e ao mesmo tempo apresentando um módulo de ruptura ligeiramente superior. Devido à abundância de ossos bovinos na forma de resíduos no Brasil o OBC pode ser uma fonte alternativa de fundentes para a indústria de porcelana.

\section{AGRADECIMENTOS}

À Fapesp (Projeto 03/12721-2) e ao CNPq pela concessão da bolsa de iniciação científica (Programa PIBIC).

\section{REFERÊNCIAS}

[1] P. Rado, An Introduction to the Technology of Pottery, $2^{\text {nd }}$ Ed., Pergamon Press, Oxford, Inglaterra (1988) p. 266.

[2] A. Kara, R. Stevens, J. Eur. Ceram. Soc. 22, 5 (2002) 737.

[3] A. Kara, R. Stevens, J. Eur. Ceram. Soc. 22, 5 (2002) 731. 
[4] W. M. Carty, U. Senapati, J. Am. Ceram. Soc. 81, 1 (1998) 3.

[5] M. G. Fonseca, G. R. Paula, R. A. Teixeira, F. G. Melchiades, A. O. Boschi, in Anais do $43^{\circ}$ Congresso Brasileiro de Cerâmica (1999) 44301-44311.

[6] S. A. F. Batista, P. F. Messer, R. J. Hand, Brit. Ceram. Trans. 100, 6 (2001) 256.
[7] P. D. S. S. Pierre, J. Am. Ceram. Soc. 37, 6 (1954) 243. [8] P. D. S. S. Pierre, J. Am. Ceram. Soc. 38, 6 (1955) 217. [9] P. D. S. S. Pierre, J. Am. Ceram. Soc. 39, 4 (1956) 147. [10] R. Y. Miyahara, D. Gouvêa, S. M. Toffoli, Cerâmica 53, 327 (2007) 234.

[11] D. Gouvêa, S. Bernard, G. A. V. Alatrista, S. M. Tofolli, Cerâmica 53, 328 (2007) 423.

(Rec. 27/06/2008, Ac. 15/01/2009) 